\title{
Proceeding
}

Supplementary Issue: Rio 2016 Olympic Games Second Anniversary Special Edition. Olympic Studies Forum, 2-3 October 2018. Federal University of Espirito Santo, (Vitória - Espirito Santo), Brazil

\section{The Globall Football Glossary® Application: Timeless product and legacy of the 2014 FIFA World Cup ${ }^{\mathrm{TM}}$}

NELSON TODT1,2 , CRISTINA PERNA ${ }^{1,3}$, HELOÍSA DELGADO ${ }^{1,3}$, EDUARDO PELLANDA ${ }^{4}$, DIEGO FREITAS FURTADOD ${ }^{1,4}$, LUIS HENRIQUE ROLIM ${ }^{1,5}$

${ }^{1}$ Olympic Studies Research Group, Pontifical Catholic University of Rio Grande do Sul (PUCRS), Porto Alegre, Brazil

${ }^{2}$ School of Health Sciences (Physical Education), Pontifical Catholic University of Rio Grande do Sul (PUCRS), Porto Alegre, Brazil

${ }^{3}$ School of Humanities (Letters), Pontifical Catholic University of Rio Grande do Sul (PUCRS), Porto Alegre, Brazil

${ }^{4}$ School of Communication, Arts and Design, Pontifical Catholic University of Rio Grande do Sul (PUCRS), Porto Alegre, Brazil

${ }^{5}$ German Sports University Cologne, Cologne, Germany

\begin{abstract}
This paper aims to analyse the development process of the Globall Football Glossary®. The Globall Football Glossary $\circledR$ is a mobile application developed by the Olympic Studies Research Group (GPEO) of the Pontifical University Catholic of Rio Grande do Sul (PUCRS) for the FIFA World Cup Brazil 2014, aiming to broaden the cultural exchange among supporters and professionals during the megaevent. The application has more than 300 football terms and makes them available in four languages: Spanish, English, Portuguese and Russian. In the first development stage of the application it was used the different methodological steps of the Terminology's Communication Theory for the definition of its database. As a result, it was created the Football Multilingual Lexicon containing eight semantical fields related to football: arbitration, areas of the field, equipment, act/action, positions, tactics, people e terms of the game. In the second development stage, the use of the terminotics and the concept Learning by Doing resulted in the creation and update of the
\end{abstract}

Corresponding author. Rua Silvio Silveira Soares, 2406 casa 117. CEP: 91910-460. Porto Alegre, Brazil.

E-mail: nelson.todt@pucrs.br

Supplementary Issue: Rio 2016 Olympic Games Second Anniversary Special Edition. Olympic Studies Forum, 2-3 October 2018. Federal University of Espírito Santo, (Vitória - Espírito Santo), Brazil.

JOURNAL OF HUMAN SPORT \& EXERCISE ISSN 1988-5202

(C) Faculty of Education. University of Alicante

doi:10.14198/jhse.2019.14.Proc3.11 
application. A hybrid application was the choice of the authors made through an online platform for the development of applications called Thunkable. In this sense, the Globall Football Glossary ${ }^{\circledR}$ is characterized as a product that contributed with the academic-scientific legacy of the FIFA World Cup Brazil 2014 as well as timeless since it is updated every edition of the megaevent. Keywords: Football; Glossary; Application; Learning by doing; World Cup; Legacy.

\section{Cite this article as:}

Todt, N., Perna, C., Delgado, H., Pellanda, E., Furtadod, D.F., \& Rolim, L.H. (2019). The Globall Football Glossary ${ }^{\circledR}$ Application: Timeless product and legacy of the 2014 FIFA World Cup ${ }^{\mathrm{TM}}$ (in Portuguese). Journal of Human Sport and Exercise, 14(3proc), S381-S390. doi:https://doi.org/10.14198/ihse.2019.14.Proc3.11 


\section{INTRODUÇÃO}

A Copa do Mundo de Futebol é considerada um megaevento esportivo pelo seu enfoque além da organização da competição, ou seja, no desenvolvimento de estruturas físicas (estádios, centros de treinamentos, etc.) e no remodelamento-comercialização do espaço urbano visando deixar um legado para a população local (De Paula, 2014). Nesse sentido, megaeventos são caracterizados como negócios complexos provenientes de objetivos socioeconômicos que refletem em volumosos investimentos para os países, os estados e as cidades sede (Preuss, 2008). Como consequência desses megaeventos, iniciativas de cunho sociocultural e acadêmico-científica também são geradas, fazendo parte do rol de legados que fogem da análise econômica de custo-benefício (Tavares, 2011). A fim de colaborar com essa forma de legado para a Copa do Mundo de Futebol da FIFA de 2014, megaevento realizado no Brasil, e visando expandir o intercâmbio cultural entre torcedores e profissionais, o Grupo de Pesquisa em Estudos Olímpicos (GPEO) da Pontifícia Universidade Católica do Rio Grande do Sul (PUCRS) criou o Globall Football Glossary ${ }^{\circledR}$. 0 aplicativo mobile disponibiliza mais de 300 termos e torna multilíngue 0 acesso ao vocabulário do futebol em quatro idiomas: Espanhol, Inglês, Português e Russo (este último incluído na última versão devido a realização da Copa do Mundo de Futebol FIFA na Rússia em 2018).

É importante salientar que a elaboração de um produto terminográfico é uma tarefa complexa e meticulosa, que exige o uso de conceitos provenientes da Terminologia e dos Estudos da Tradução. Com base na Teoria Comunicativa da Terminologia, os termos são definidos como unidades complexas que fazem parte das línguas naturais, além de serem polissêmicos, não normalizados e com as seguintes características: (i) possuem significados adquiridos por unidades lexicais dentro de um contexto de comunicação especializada; (ii) devem ser estudados dentro de seu contexto; (iii) residem na situação comunicacional onde são empregados, na especialização de suas características semânticas, no conhecimento que representam e no tipo de significação (Adelstein \& Cabré, 2002). A Terminologia e a Tradução possuem vários pontos em comum, mas seus objetos de estudo, pressupostos teóricos e metodologias são bastante diferenciados, caracterizando-se como disciplinas autônomas. Enquanto a Terminologia constitui um fim em si mesma, apresentando um caráter finalista, a Tradução constitui apenas um meio para realizar outras atividades de caráter linguístico, tais como a tradução, a interpretação e a produção de textos especializados (Ramos, 2001). Entretanto, a relação existente entre a Terminologia e a Tradução, deve ser considerada positiva já que as pesquisas terminológicas proporcionam ao tradutor não somente os materiais terminográficos de que precisa para bem realizar seu trabalho, mas, também, e principalmente, "0 conhecimento aprofundado sobre o funcionamento dessas linguagens e os recursos que podem ser utilizados para a otimização desse trabalho" (Ramos, 2001, p. 170).

Outra abordagem determinante neste trabalho é a terminótica que, segundo Gouadec (1992), é um conjunto de situações e atividades nas quais a Informática intervém para construir, organizar, administrar ou explorar metodologias. Assim, a terminótica é considerada um instrumento para avançar os idiomas e pesquisas terminológicas no sentido das "novas tecnologias da informação, vinculadas, por um lado, à Informática e, por outro, aos resultados dos estudos sistemáticos da língua" (Cabré, 1993, p. 373), além de permitirem a produção e comercialização de produtos terminográficos.

Para o desenvolvimento do produto final proposto por este trabalho, optou-se pelo método Learning by Doing (traduzido livremente como "aprender fazendo"). Este é um método que propõe uma nova perspectiva quanto ao aprendizado. Esse approach está diretamente relacionado ao autodesenvolvimento. Suas técnicas envolvem o ensino e aprendizado através de atividades empíricas que geram conhecimento. 0 método Learning by Doing vem sendo utilizado em diversas áreas, tais como ensino de idiomas modernos, 
treinamentos empresariais, e no ensino relacionado às ciências físicas e matemáticas (Bot et al., 2005). 0 Globall Football Glossary ${ }^{\circledR}$, em sua fase de desenvolvimento e atualização do aplicativo mobile, teve base nesse método.

Desde sua criação, em 2014, o Globall Football Glossary ${ }^{\circledR}$ se destacou enquanto produto tecnológico inovador no âmbito do mercado brasileiro, ou seja, como um aplicativo desenvolvido para distribuição gratuita, visando uma maior disseminação, padronização e adequação da terminologia utilizada no âmbito do Futebol. $O$ aplicativo é fruto de um trabalho interdisciplinar e pode ser considerado singular entre os meios informativos (rádio e televisão), pois possibilita acesso fácil e rápido a diferentes termos do Futebol, colaborando para 0 trabalho de profissionais que socializam este tipo de conhecimento (tradutores, intérpretes, revisores) e, também, de não profissionais, facilitando o entendimento do público leigo em quatro idiomas diferentes. Dessa forma, esse artigo tem como objetivo geral analisar o processo de desenvolvimento do Globall Football Glossary ${ }^{\circledR}$. Os objetivos específicos deste artigo serão divididos em duas etapas de análise: (1) o processo de definição dos termos que formam o corpus do Globall Football Glossary ${ }^{\circledR}$, isto é, a metodologia na elaboração do produto terminográfico adequado à cultura local dos idiomas; (2) a aplicação da terminótica (terminologia + informática) e do conceito Learning by Doing no desenvolvimento e atualização do aplicativo.

\section{METODOLOGIA}

\section{Football Multilingual Lexicon}

Antes de 0 aplicativo mobile Globall Football Glossary ${ }^{\circledR}$ ser desenvolvido, houve uma primeira fase de criação para a definição dos termos que formariam o seu corpus. Foi elaborado um produto terminográfico chamado Football Multilingual Lexicon (Todt et al., 2012; Perna et al., 2013). Para a elaboração desse produto seguiram-se sete etapas metodológicas baseadas na Teoria Comunicativa da Terminologia (Almeida, 2000):

1. Definição do projeto: para decidir sobre o âmbito do conhecimento, o tema, o enfoque e a organização que o apoiou;

2. Redação do plano de pesquisa: para definir itens básicos, tais como (i) o tipo de produto terminográfico que será elaborado (glossário, léxico, dicionário, etc.) e suas características; (ii) a organização da equipe que elaborou o projeto(que contou com três conhecimentos básicas: sobre o tema; sobre um ou mais idiomas que envolvem o produto e; sobre a metodologia); (iii) a infraestrutura informatizada (dois computadores e um sistema de gestão de base de dados); e (iv) o período de tempo que a equipe levará para a finalização do projeto;

3. Início da pesquisa: dividida em três momentos: (i) seleção do material (textos, dicionários, etc.) necessário que inclui o vocabulário pertinente ao tema escolhido; (ii) elaboração da estrutura do conteúdo a fim de facilitar a delimitação da área temática e a classificação e tratamento das unidades terminológicas; (iii) seleção dos textos (corpus) de referência do tema escolhido.

4. Elaboração da base de dados: envolvendo os seguintes passos: (i) definição do programa de tratamento de textos e base de dados que será utilizado; (ii) definição das categorias as quais os termos se inserem (campos semânticos), em função das características do produto terminográfico e; (iii) definição das unidades terminológicas (no caso de dois termos para definir a mesma ação, escolher qual termo será utilizado); 
5. Revisão da base de dados: revisão detalhada do produto terminográfico, a fim de detectar possíveis falhas e corrigi-las, seguindo as normas que envolvem a produção de dicionários/léxicos.

6. Elaboração do protótipo operacional: criação de uma plataforma virtual, a partir da base de dados escolhida, para armazenamento dos termos e que possibilite acesso do público ao conteúdo.

7. Edição do conteúdo: buscar contextos reais aos quais se aplicam cada um dos termos (nos diferentes idiomas que compõe o léxico) a partir da participação de especialistas na área do futebol, para que os termos estivessem de acordo com a terminologia utilizada nas obras de referência da área futebolística.

\section{Globall Football Glossary ${ }^{\circledR}$}

A segunda fase do projeto é caracterizada pelo desenvolvimento do aplicativo Globall Football Glossary ${ }^{\circledR}$ como produto final, tanto na criação em 2014 como na atualização em 2018. Esse processo teve como base a metodologia da terminótica (terminologia + informática) e, a fim de buscar qualidade e qualificação técnicoprofissional para a criação desse produto, foi realizada uma parceria com o Laboratório de Pesquisa em Mobilidade e Convergência Midiática da Pontifícia Universidade Católica do Rio Grande do Sul (UBILABPUCRS). Através do conceito Learning by Doing, explorou-se novas tecnologias no cenário da comunicação e da mobilidade. De acordo com Bolt et al. (2005), no âmbito educacional o processo de Learning by Doing pode ser divido em quatro etapas, similares ao processo de resolução de problemas: (1) técnica (envolvendo o entendimento e avaliação dos recursos disponíveis antes de resolver uma situação-problema); (2) indutiva (em face a situação-problema, hipóteses são feitas e um novo processo de raciocínio deverá ser induzido com a ajuda da intuição para possíveis soluções); (3) dedutiva (pensando na experimentação e validação, consequências deverão ser deduzidas das hipóteses formuladas na fase anterior) e; (4) aplicativa (entendimento do potencial fornecido pelo conhecimento novo e a possibilidade provocar mudanças no resultados).

O processo de construção e evolução-aprimoramento do Globall Football Glossary ${ }^{\circledR}$ envolveu variações, como sistemas operacionais e dispositivos. Desta forma, além de definirmos os objetivos do produto e seu público-alvo, os requisitos técnicos passaram a ser fundamentais para uma escolha coerente da plataforma a ser utilizada, pois para cada sistema operacional, seja no smartphone ou tablet, existe uma plataforma específica para seu desenvolvimento. Assim, primeiramente, foi necessário identificar se a plataforma tem a funcionalidade de Gerenciamento de Aplicativos Móveis (MAM) e as características de Gerenciamento de Dispositivos Móveis (MDM). Essas características tratam de questões como controle de acesso centralizado e gerenciamento de versão do aplicativo. A plataforma também deveria incluir outras características importantes, como: recursos abrangentes de testes, codificação amigável móvel, protocolos de transferência de dados, armazenamento seguro on device e capacidades de identificação de rede.

Outra questão muito importante foi se o aplicativo seria web App, nativo ou híbrido. Web App não é um aplicativo real e não fica disponível nas lojas de aplicativos (ex: Apple Store e Play Store). O Web App é um site desenvolvido exclusivamente para dispositivos móveis que possui uma programação que reconhece e se adapta a um smartphone ou tablete. É uma forma de baixo custo e de fácil desenvolvimento em comparação com os aplicativos híbridos e nativos e toda sua programação é feita utilizando HTML5, Cascading Style Sheets (CSS) e Javascript. Seu acesso pode se dar de qualquer sistema operacional, desde que o mesmo possua um navegador web instalado no dispositivo (Hartmann, Stead \& Degani, 2011). 
Os aplicativos nativos são softwares autônomos instalados diretamente, e com linguagem exclusiva, em um sistema operacional a partir da loja de aplicativos (Apple Store - iOS; Play Store - Android). Eles são desenvolvidos para uma plataforma única em sua linguagem de programação nativa (Swift - iOS; Java Android). Por serem programados exclusivamente, 0 aplicativo nativo é mais rápido e com mais funções que o Web App, como acesso a câmera, GPS, notificações push e podem funcionar sem conexão com a internet. Porém, para desenvolver um aplicativo nativo é necessário seguir guias padrões de design e funcionalidade de cada sistema operacional, o que torna o processo mais trabalhoso do que com o Web App (Madureira, 2017).

O aplicativo híbrido é uma mescla entre o Web App e o nativo e é construído na linguagem HTML5, CSS e Javascript, assim como o Web App, então, apenas uma parte do código nativo é escrito e o aplicativo fica disponível para download nas App Stores. Da mesma forma como o aplicativo nativo, o híbrido apresenta um custo de manutenção nas App Stores. $O$ aplicativo híbrido é mais simples e rápido de ser desenvolvido do que o nativo, porém, ele será dependente de uma conexão com a internet (Da Silva \& Santos, 2014).

Optou-se por um aplicativo híbrido, desenvolvido em grande parte, em HTML5, utilizando tecnologia Web App inserido dentro de um invólucro de aplicativo nativo. Isso permite que o aplicativo tenha a funcionalidade de um aplicativo nativo e também reduz o esforço de desenvolvimento ao criar versões para plataformas adicionais.

Através da metodologia Learning by Doing (um processo essencialmente prático), o aplicativo Globall Football Glossary ${ }^{\circledR}$ foi desenvolvido através de uma plataforma online para desenvolvimento de aplicativos chamado Thunkable. Nela é possível gerar, através de uma única programação, aplicativos tanto para IOS quanto para Android, sistemas que utilizam duas linguagens completamente diferentes. Além disso, utiliza princípios básicos de programação sem exigir o conhecimento de uma linguagem específica, tornando uma ferramenta de fácil acesso para pessoas que não especialistas na área de desenvolvimento de aplicativos. O Thunkable é totalmente voltado para educação e não tem fins lucrativos. Sendo assim, o Thunkable se tornou uma peça fundamental para o desenvolvimento do Globall Football Glossary ${ }^{\circledR}$, reduzindo o tempo de programação e a necessidade de um conhecimento específico em determinada linguagem.

\section{RESULTADOS}

\section{Football Multilingual Lexicon}

Com base nas sete etapas metodológicas baseadas na Teoria Comunicativa da Terminologia (Almeida, 2000), o Football Multilingual Lexicon, conta com 300 termos divididos em 8 campos semânticos relacionados ao futebol. São eles: (1) arbitragem (incluindo termos que são relacionados aos oficiais da partida e às regras do jogo); (2) áreas do campo (referente as delimitações que compõem o campo de jogo); (3) equipamentos (todos os materiais esportivos que fazem parte do esporte); (4) ato (ações que fazem parte do esporte, ex: diferentes dribles e jogadas); (5) posições (posicionamentos dos jogadores que compõe a escalação da equipe); (6) táticas (estratégias utilizadas pela equipe/treinador); (7) pessoal (indivíduos que exercem diferentes funções no futebol, ex: médico, gadula, massagista, etc) e; (8) termos do jogo (expressões variadas que não se encaixam nos outros campos semânticos).

Utilizou-se para a montagem do corpus os comandos manuais avançados do Google e cursos disponibilizados pelo Google News Lab (https://gweb-newslab-qa.appspot.com/). Tais comandos e cursos foram utilizados tanto para a busca de definições quanto de contextos (e para virtualmente qualquer outro propósito que envolvesse a pesquisa online). Estes também serviram para checar se realmente uma dada 
expressão é utilizada em um contexto específico. As definições utilizadas vieram, em sua maioria, de glossários online especializados em futebol. Embora tenham sido checadas a confiabilidade e a reputação tanto dos autores dos glossários quanto dos sites onde tais glossários tenham sido encontrados, o uso dos comandos manuais avançados garantiu que os termos eram realmente relevantes e utilizados no mundo do futebol. De maneira geral, deu-se preferência a contextos em sites de instituições futebolísticas (tais como FIFA, UEFA e CBF) e a sites especializados e/ou com departamentos especializados no assunto (como, por exemplo, periódicos de futebol ou periódicos gerais com colunas dedicadas a este esporte).

Após essa seleção e divisão de termos em português brasileiro (em função de ser o país que sediou a copa naquele ano), a primeira versão (voltada para a Copa do Mundo de Futebol FIFA 2014) foi enviada para especialistas em futebol que dominassem um dos seguintes idiomas: inglês britânico (visto que a Inglaterra foi o país que originou esse esporte), o espanhol da Espanha (em virtude da parceria com a Universidad Europea de Madrid). Para a atualização do aplicativo em 2018, houve a inclusão do idioma russo e 0 processo metodológico foi realizado em parceria com a Russian International Olympic University.

A tradução dos termos não foi feita de forma literal. Os especialistas encarregados dessa tradução buscaram expressões referentes a cada uma das definições no seu idioma de domínio (ex: carrinho não foi traduzido como "little car", mas sim, como slide tackle, expressão utilizada para esse ato em inglês). Com o glossário de termos em três idiomas, foram desenvolvidos novos resultados: como a apresentação da definição dos termos (ex: carrinho é um lance em que o jogador se atira no chão, projetando as pernas à frente e, em geral o objetivo é atingir a bola no menor tempo possível) e citações de jornais e revistas especializadas em futebol apresentando cada termo em diferentes contextos reais (ex: Lucas chega no carrinho e derruba tanto Cleverley, quanto Welbeck na meia-lua da área do Liverpool).

\section{Globall Football Glossary ${ }^{\circledR}$}

Para o desenvolvimento do aplicativo, utilizamos a plataforma de criação de aplicativos Thunkable, a partir dela criamos o modelo de aplicativo ideal, respeitando tecnicamente as diferenças entre os dois sistemas operacionais (Android e iOS). Porém, ao decorrer desse processo esbarramos em questões técnicas. Entre elas estava o limite de telas permitido, pois havíamos criado muitas telas com formatos diferentes, o que fez com que 0 aplicativo travasse e não fosse possível dar continuidade a programação. Nesse sentido, aproveitou-se o ocorrido como aprendizado para mudar a estrutura e redefinir 0 aplicativo, pois 0 objetivo era ter algo funcional.

Baseado na metodologia Learning by Doing, recomeçamos o aplicativo e redesenhamos a organização das telas e programação com o objetivo de usar o menor número de telas possíveis, deixando o Globall Football Glossary ${ }^{\circledR}$ mais funcional através de um sistema que armazena todas as informações em uma única tela.

Para o desenvolvimento do aplicativo foi necessário também a elaboração de uma logomarca (Imagem 1) e identidade visual do produto, que foi desenvolvido pela Assessoria de Comunicação e Marketing da Pontifícia Universidade Católica do Rio Grande do Sul (ASCOM-PUCRS).

O próximo desafio, dada a apresentação final do produto, foi o lançamento antes da Copa do Mundo de Futebol FIFA 2014. Durante o desenvolvimento do aplicativo, encaminharam-se os pedidos para registro de software e de marca através do Instituto Nacional da Propriedade Industrial (INPI) do Brasil. 0 trabalho obteve a Patente de Programa de Computador (n BR512015001125-0 de: 11/06/2014), o registro de Marca Registrada de Produto ( $n^{\circ}$ PI907789480) e Marca Registrada de Serviço ( $n^{0}$ PI907755259), todos pelo Instituto Nacional da Propriedade Industrial (INPI) do Brasil. 


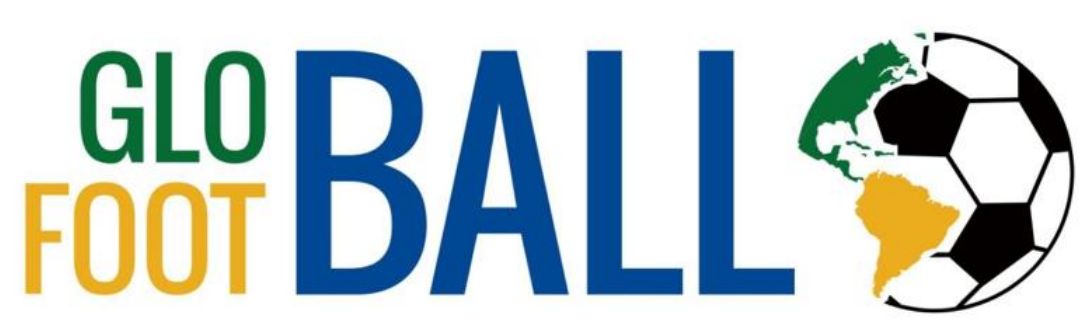

Imagem 1. Logomarca Globall Football Glossary.

Com o registro da marca, foi lançado em 11 de junho de 2014 (dia anterior à abertura da Copa do Mundo de Futebol FIFA 2014), o Globall Football Glossary ${ }^{\circledR}$. O produto foi distribuído para download gratuito. Vale ressaltar que o projeto de desenvolvimento do aplicativo, teve apoio da Fundação de Amparo à pesquisa do Estado do Rio Grande do Sul (FAPERGS), do Conselho Nacional de Desenvolvimento Científico e Tecnológico (CNPq) e do Programa de Apoio à Integração entre Áreas (PRAIAS) da PUCRS.

\section{CONSIDERAÇÕES FINAIS}

O Globall Football Glossary ${ }^{\circledR}$ é um produto que colabora com o legado acadêmico-científico da Copa do Mundo de Futebol FIFA 2014, realizada no Brasil. 0 mesmo passou por uma atualização importante em virtude da Copa do Mundo de Futebol FIFA 2018, realizada na Rússia, o que marca o seu desenvolvimento como uma construção atemporal, já que vem se renovando a cada edição do megaevento.

A construção de um aplicativo fruto da realização de megaeventos esportivos deve ter caráter internacional. Dessa forma, o aplicativo Globall Football Glossary ${ }^{\circledR}$ alinhou-se à ideia de dinamizar ações propostas pelos diferentes setores da Pontifícia Universidade Católica do Rio Grande do Sul e da Comunidade Nacional e Internacional. Neste âmbito, o Grupo de Pesquisa em Estudos Olímpicos da PUCRS teve como parceiros 0 Laboratório de Pesquisa em Mobilidade e Convergência Midiática (UBILAB) da PUCRS, a Universidad Europea de Madrid e a Russian International Olympic University.

As limitações que apareceram ao longo do desenvolvimento do aplicativo exigiram que a equipe de criação do mesmo aprendesse com os erros e se baseasse na experiência adquirida a fim de alcançar melhores resultados. A metodologia Learning by Doing foi fundamental para esse exercício de aprendizagem, através do estímulo ao pensamento crítico e associativo sobre o próprio trabalho.

Ainda são limitados os dados e informações sobre os downloads do aplicativo. A partir de uma análise desses dados poderemos compreender a praticabilidade e 0 alcance do Globall Football Glossary ${ }^{\circledR}$, além de avaliar a integridade geral do aplicativo a partir de tendências de dados, tais como: aquisição, público, comportamento e conversões.

Para a Copa do Mundo de Futebol FIFA 2022, que será realizada no Catar, pretende-se ampliar o Globall Football Glossary ${ }^{\circledR}$ incluindo o idioma árabe. Entretanto, para proporcionar uma maior visibilidade do produto, seria interessante que o aplicativo fizesse parte do planejamento estratégico de marketing da Copa do Mundo FIFA, sendo incluído como um dos produtos oficiais do evento. 


\section{REFERÊNCIAS}

Adelstein, A., \& Cabré, M. T. (2002). The specificity of units with specialized meaning: polysemy as explanatory factor. DELTA: Documentação de Estudos em Lingüística Teórica e Aplicada, 18(SPE), pp. 1-25. https://doi.org/10.1590/s0102-44502002000300003

Almeida, G. M. B. (2000). Teoria Comunicativa da Terminologia (TCT): Uma aplicação [Communicative Theory of the Terminology: An application] (Doctoral Dissertation). FCLAR-UNESP, Brazil.

Bot, L., Gossiaux, P. B., Rauch, C. P., \& Tabiou, S. (2005). 'Learning By Doing': A teaching method for active learning in scientific graduate education, European Journal of Engineering Education, 30(1), pp. 105-119. https://doi.org/10.1080/03043790512331313868

Cabré, M. T. (1993). La Terminologia: Teoria, Metodologia, Aplicaciones [The Terminology: Theory, Methodology and Applications]. Barcelona: Editorial Antártida/Empúries. https://doi.org/10.7202/002110ar

De Paula, M. (2014). The 2014 World Cup in Brazil: Its legacy and challenges, Human Rights programme coordinator for the Heinrich Böll Foundation in Brazil. https://doi.org/10.1163/2210-7975_hrd-9951$\underline{0029}$

Da Silva, M. M., \& Santos, M. T. P. (2014). Os Paradigmas de Desenvolvimento de Aplicativos para Aparelhos Celulares [The Paradigms of Application Development for Mobiles], Revista T.I.S., 3(2), pp. 162-170. Retrieved from http://www.revistatis.dc.ufscar.br/index.php/revista/article/view/86/80

Gouadec, D., \& Université d'automne en terminologie. (1992). Terminologie et terminotique: Outils, modèles et méthodes [Terminology and Terminotics: Tools, Models and Methods], Actes de la première Université d'automne en terminologie, Rennes 2, 21 au 26 septembre 1992. Paris: Maison du dictionnaire. https://doi.org/10.7202/002128ar

Hartmann, G., Stead, G., \& DeGani, A. (2011). Cross-platform mobile development, Mobile Learning Environment, Cambridge, 16(9), pp. 158-171. Retrieved from https://wss.apan.org/jko/mole/Shared\%20Documents/CrossPlatform\%20Mobile\%20Development.pdf

Madureira, D. (2017, March 8). Aplicativo nativo, web App ou aplicativo híbrido [Native Application, web App or hybrid application]. Retrieved on December 18, 2018, from https://usemobile.com.br/aplicativo-nativo-web-hibrido/

Preuss, H. (2008). Aspectos Sociais dos Megaeventos Esportivos [Social Aspects of the Sporting Megaevents]. In Rubio, K. (Ed.), Megaeventos Esportivos, Legado e Responsabilidade Social (pp. 13-35). São Paulo, Brazil: Casa do Psicólogo. https://doi.org/10.5007/2175-8042.2009n32-33p71

Perna, C., Delgado, H., Todt, N. S., Simón, J., Salvador, L., Rollsing, L. Z., \& Ligocky, F. (2013). Football Multilingual Lexicon: A legacy of the FIFA 2014 World Cup in Brazil. In Olympic Legacies: Impacts of mega-events on cities (pp. 66-66). London, England: University of East London.

Ramos, P. C. (2001). Interface Tradução Terminologia [Terminology Translation Interface]. In Kriegerm M. G., \& Maciel, A. M. B. (Eds.), Temas de Terminologia (pp. 164-170). Porto Alegre/São Paulo, Brazil: Ed. Universidade/UFRGS/Humanitas/USP.

Tavares, O. (2011). Megaeventos Esportivos [Sporting Megaevents], Revista Movimento, 17(3), pp. 11 35. https://doi.org/10.22456/1982-8918.23176

Todt, N. S., Perna, C., Delgado, H., Sá, R. N., Dubois, G. C., \& Silva, A. C. O. (2012). Football Multilingual Lexicon: A legacy of the FIFA 2014 World Cup in Brazil. In International Convention on Science, Education and Medicine in Sport, 2012, Glasgow. (pp. 290-291). London, England: Routledge. 


\section{(c) $\$$ (i) $\ominus$}

This work is licensed under a Attribution-NonCommercial-NoDerivatives 4.0 International (CC BY-NC-ND 4.0). 